УДК 517.986.7

\title{
Parameter Determination in a Differential Equation of Fractional Order with Riemann-Liouville Fractional Derivative in a Hilbert Space
}

\author{
Dmitry G. Orlovsky* \\ National Research Nuclear University MEPhI \\ Kashirskoye shosse, 31, Moscow, 115409 \\ Russia
}

Received 10.10.2014, received in revised form 20.11.2014, accepted 15.12.2014

The Cauchy type problem for a differential equation with fractional derivative and self-adjoint operator in a Hilbert space is considered. The problem of parameter determination in equation by the value of the solution at a fixed point is presented. Theorems of existence and uniqueness of the solution are proved.

Keywords: equation of fractional order, Hilbert space, self-adjoint operator, Cauchy-type problem, MittagLeffler function, inverse problem, characteristic function.

\section{Introduction}

In recent years, theory of differential equations with fractional derivatives has attracted considerable interest. This is primarily due to the application of this theory to new models of physical processes. However the theory has been developed for some time. The first steps were made by L. Euler, P. Laplace and J. Furier. Further development of this theory was associated with the works of N.H. Abel, J. Liouville and B. Riemann. An important advancement in the development of the theory is presented in [1-4].

One of the modern methods for studying equations of mathematical physics is the theory of differential equations in Banach and Hilbert spaces. Abstract differential equations of fractional order are considered in [5-8].

Let us consider a closed linear operator $A$ in a Banach space $H$. The operator domain $D(A)$ is dense and $0<\alpha<1, u_{0} \in H$. Consider the following Cauchy-type problem

$$
\begin{gathered}
D^{\alpha} u(t)=A u(t)+f(t), t>0, \\
\lim _{t \rightarrow 0} D^{\alpha-1} u(t)=u_{0},
\end{gathered}
$$

where $D^{\alpha}$ is the Riemann-Liouville fractional derivative of order $\alpha$ :

$$
\begin{aligned}
& \left(D^{\alpha} u\right)(t)=\frac{1}{\Gamma(1-\alpha)} \frac{d}{d t} \int_{0}^{t} \frac{u(s)}{(t-s)^{\alpha}} d s \\
& \left(D^{\alpha-1} u\right)(t)=\frac{1}{\Gamma(1-\alpha)} \int_{0}^{t} \frac{u(s)}{(t-s)^{\alpha}} d s
\end{aligned}
$$

*odg@bk.ru

(c) Siberian Federal University. All rights reserved 
and $\Gamma$ is the Gamma function.

A solution of (1), (2) is a continuous function $u(t)$ in $H$ for $t>0$ such that $D^{\alpha-1} u(t)$ is continuously differentiable in $H$ for $t>0$. Function $u(t)$ takes its values in $D(A)$ and satisfies $(1),(2)([7])$.

Along with problem (1), (2), let us consider the homogeneous problem

$$
\begin{gathered}
D^{\alpha} u(t)=A u(t), t>0, \\
\lim _{t \rightarrow 0} D^{\alpha-1} u(t)=u_{0} .
\end{gathered}
$$

We say that the problems (1), (2) and (3), (4) are uniformly well-posed ( [7]) if there is operator function $V(t)$ (solution operator) that commutes with A. There are also two constants $M>0$ and $\omega \in R$ such that for every $u_{0} \in D(A)$ function $V(t) u_{0}$ is the only solution of problem (3), (4) and for any $t>0$ the following estimate

$$
\|V(t)\| \leqslant M t^{\alpha-1} e^{\omega t}
$$

is valid.

If space $H$ has the Radon-Nikodym property (every absolutely continuous function represented by the integral of its derivative) then problem (3), (4) is uniformly well-posed if and only if when $\operatorname{Re} \lambda>\omega$ the operator $A$ has the resolvent $R\left(\lambda^{\alpha}\right)=\left(\lambda^{\alpha} I-A\right)^{-1}$. The resolvent satisfies the following inequality [9]

$$
\left\|\frac{d^{n} R\left(\lambda^{\alpha}\right)}{d \lambda^{n}}\right\| \leqslant \frac{M \Gamma(n+\alpha)}{(\operatorname{Re} \lambda-\omega)^{n+\alpha}} .
$$

In this case the solution operator has the following representation [9]

$$
V(t)=D^{1-\alpha} \frac{1}{2 \pi i} \text { v.p. } \int_{\omega_{0}-i \infty}^{\omega_{0}+i \infty} \lambda^{\alpha-1} e^{\lambda t} R\left(\lambda^{\alpha}\right) d \lambda \quad\left(\omega_{0}>\max (0, \omega)\right) .
$$

Let us consider the Cauchy type problem (1), (2) assuming that problem is uniformly wellposed. The solvability of this problem can be proved if

(C1) functions $f(t)$ and $A f(t)$ are continuous for $t>0$ in $H$ and absolutely integrable at $t=0$;

(C2) function $D^{\alpha-1} f(t)$ is continuous for $t \geqslant 0$ and continuously differentiable for $t>0$, function $D^{\alpha} f(t)$ is absolutely integrable at $t=0$.

If any of these two conditions is satisfied then the solution of problem (1), (2) exists. The solution is unique and it is given by the formula [9]

$$
u(t)=V(t) u_{0}+\int_{0}^{t} V(t-s) f(s) d s .
$$

The principle of subordination is one of the important properties of equations with fractional derivatives. Along with problem (3), (4) we consider another Cauchy type problem with other differentiation order $\beta(\alpha \leqslant \beta \leqslant 1)$

$$
\begin{gathered}
D^{\beta} u(t)=A u(t), t>0, \\
\lim _{t \rightarrow 0} D^{\beta-1} u(t)=u_{0} . \\
-56-
\end{gathered}
$$


We also consider function $f_{\tau, \nu}(t)$ defined by the equality

$$
f_{\tau, \nu}(t)=\left\{\begin{array}{l}
\frac{1}{2 \pi i} \int_{\substack{\sigma-i \infty \\
t<0}}^{\sigma+i \infty} \exp \left(t z-\tau z^{\nu}\right) d z, t \geqslant 0 \\
0, t \geq 0
\end{array}\right.
$$

where $\sigma>0, \tau>0,0<\nu<1$ and branch $z^{\nu}$ is selected such that $\operatorname{Re} z^{\nu}>0$ for $\operatorname{Re} z>0$. This function is non-negative ( [10] and [11])

$$
\int_{0}^{\infty} f_{\tau, \nu}(t) e^{\lambda \tau} d \tau=t^{\nu-1} E_{\nu, \nu}\left(\lambda t^{\nu}\right)
$$

where $E_{\alpha, \beta}(x)$ is the generalized Mittag-Leffler function

$$
E_{\alpha, \beta}(x)=\sum_{n=0}^{\infty} \frac{x^{n}}{\Gamma(\alpha n+\beta)} .
$$

The principle of subordination ( [11]) states that if problem (7), (8) is uniformly well-posed then problem $(3),(4)$ is also uniformly well-posed. Moreover, the solution operator $V(t)$ of problem $(3),(4)$ is expressed in terms of the solution operator $U(t)$ of problem $(7),(8)$ by the following relation

where $\nu=\alpha / \beta$.

$$
V(t)=\int_{0}^{\infty} f_{\tau, \nu}(t) U(\tau) d \tau
$$

Throughout this paper it is assumed that the space $H$ is a Hilbert space and operator $A$ is self-adjoint and non-positive. Any reflexive Banach space has the Radon-Nikodym property so in our case this property is satisfied.

Lemma 1. Let us assume that $H$ is a Hilbert space, operator $A$ is self-adjoint and bounded from above, element $u_{0} \in D(A)$ and one of the conditions (C1) or (C2) is satisfied. Then the solution of Cauchy type problem (1), (2) exists, it is unique and has the form

$$
u(t)=t^{\alpha-1} E_{\alpha, \alpha}\left(t^{\alpha} A\right) u_{0}+\int_{0}^{t}(t-s)^{\alpha-1} E_{\alpha, \alpha}\left((t-s)^{\alpha} A\right) f(s) d s .
$$

Proof. It is known that any self-adjoint bounded from above operator is a generator of a strongly continuous semigroup. This means that problem $(7),(8)$ is uniformly well-posed when $\beta=1$. Applying the principle of subordination, we obtain that problem (3), (4) is also uniformly well-posed for any $0<\alpha<1$. The solution operator of problem (7), (8) is $U(t)=\exp (A t)$, therefore by virtue of the principle of subordination (taking into account that $\nu=\alpha$ ) we have the equality

$$
V(t)=\int_{0}^{\infty} f_{\tau, \alpha}(t) \exp (A \tau) d \tau=\int_{0}^{\infty} f_{\tau, \alpha}(t)\left(\int_{-\infty}^{b} e^{\lambda \tau} d E_{\lambda}\right) d \tau=\int_{0}^{\infty} \int_{-\infty}^{b} f_{\tau, \alpha}(t) e^{\lambda \tau} d E_{\lambda} d \tau,
$$

where $E_{\lambda}$ is a spectral resolution of the operator $A$ and $b$ is its upper bound. As the integrand is non-negative function, we can change the order of integration. Taking into account equation (10), we obtain

$$
V(t)=\int_{-\infty}^{b}\left(\int_{0}^{\infty} f_{\tau, \alpha}(t) e^{\lambda \tau} d \tau\right) d E_{\lambda}=\int_{-\infty}^{b} t^{\alpha-1} E_{\alpha, \alpha}\left(\lambda t^{\alpha}\right) d E_{\lambda}=t^{\alpha-1} E_{\alpha, \alpha}\left(t^{\alpha} A\right)
$$

Relation (11) directly follows from equality (6). 


\section{Inverse problem}

Let us assume that $H$ is a Hilbert space, operator $A$ is self-adjoint and non-positive, $0<\alpha<1$, $\varphi(t)$ is a scalar non zero function and $u_{0}, u_{1} \in H$. Let us find function $u(t)$ with values in the space $\mathrm{H}$ and element $p \in H$ that satisfy the following system of equations

$$
\begin{gathered}
D^{\alpha} u(t)=A u(t)+\varphi(t) p, t>0, \\
\lim _{t \rightarrow 0} D^{\alpha-1} u(t)=u_{0}, \\
u(T)=u_{1} .
\end{gathered}
$$

where $T>0$.

According to the adopted terminology problem (12)-(14) belongs to the class of inverse problems. A review of publications on this subject can be found in [12-19]. Inverse problems for equations of fractional order in Banach spaces was considered in papers [20,21]. Similar problem was considered for the first order equation (when $\alpha=1$ ) but with a fractional integral in the additional boundary condition [22].

The behavior of the entire function

$$
F(z)=\int_{0}^{T}(T-s)^{\alpha-1} E_{\alpha, \alpha}\left(z(T-s)^{\alpha}\right) \varphi(s) d s .
$$

is the key to the solvability of problem (12)-(14).

Function (15) will be denoted the characteristic function of inverse problem (12)-(14).

Theorem 1. Let us assume that $H$ is a Hilbert space, operator $A$ is self-adjoint and non-positive, $0<\alpha<1$, function $\varphi(t) \in C[0 ; T] \cap C^{1}(0 ; T]$, it is not identically equal to zero for $t \in(0 ; T]$. The derivative of function $\varphi(t)$ is absolutely integrable at $t=0$ and the elements $u_{0}, u_{1} \in H$. The solution of problem (12)-(14) is unique if and only if point spectrum of the operator $A$ does not contain zeros of the characteristic function (15). The solution of problem (12)-(14) exists if and only if

$$
\int_{-\infty}^{0}|F(\lambda)|^{-2} d\left(E_{\lambda} h, h\right)<\infty
$$

where $E_{\lambda}$ is a spectral resolution of the operator $A$, and the element

$$
h=u_{1}-T^{\alpha-1} E_{\alpha, \alpha}\left(T^{\alpha} A\right) u_{0} .
$$

Proof. According to lemma 1 the solution of direct problem (12)-(13) is given by formula (11) where one should set $f(t)=\varphi(t) p$. Setting in this formula $\mathrm{t}=\mathrm{T}$ and taking into account (14), we obtain the following equation for the unknown element $\mathrm{p}$

$$
u_{1}=T^{\alpha-1} E_{\alpha, \alpha}\left(T^{\alpha} A\right) u_{0}+\int_{0}^{T}(T-s)^{\alpha-1} E_{\alpha, \alpha}\left((T-s)^{\alpha} A\right) \varphi(s) p d s .
$$

This equation can be written in the following form

$$
B p=h,
$$

where operator $B$ is defined by the relation

$$
B p=\int_{0}^{T}(T-s)^{\alpha-1} E_{\alpha, \alpha}\left((T-s)^{\alpha} A\right) \varphi(s) p d s
$$


and element $h$ is given by (17).

Let us transform the expression that represents the operator $B$. For this we use the spectral decomposition of the operator $A$

$$
A=\int_{-\infty}^{0} \lambda d E_{\lambda}
$$

According to the operator calculus of self-adjoint operators, we have

$$
E_{\alpha, \alpha}\left((T-s)^{\alpha} A\right)=\int_{-\infty}^{0} E_{\alpha, \alpha}\left(\lambda(T-s)^{\alpha}\right) d E_{\lambda} .
$$

Therefore

$$
B=\int_{0}^{T}\left(\int_{-\infty}^{0}(T-s)^{\alpha-1} E_{\alpha, \alpha}\left(\lambda(T-s)^{\alpha}\right) \varphi(s) d E_{\lambda}\right) d s .
$$

Since the measure $d E_{\lambda} \otimes d s$ is finite on the $(-\infty ; 0] \times[0 ; T]$ then by Fubini's theorem we have

$$
B=\int_{-\infty}^{0}\left(\int_{0}^{T}(T-s)^{\alpha-1} E_{\alpha, \alpha}\left(\lambda(T-s)^{\alpha}\right) \varphi(s) d s\right) d E_{\lambda}=\int_{-\infty}^{0} F(\lambda) d E_{\lambda}=F(A) .
$$

Therefore, the equation for the unknown element $p$ takes the form

$$
F(A) p=h .
$$

Equation (18) was studied in [23]. It was proved that the solution of this equation is unique if and only if point spectrum of the operator $A$ does not contain zeros of $F(z)$. The existence of a solution of the equation follows from condition (16). The application of this result proves theorem 1.

Theorem 2. Let us assume that $H$ is a Hilbert space, operator $A$ is self-adjoint and non-positive, $0<\alpha<1$, function $\varphi(t) \in C[0 ; T] \cap C^{1}(0 ; T]$ and its derivative is absolutely integrable at $t=0$, everywhere either $\varphi(t) \geqslant 0$ or $\varphi(t) \leqslant 0$ and $\varphi(T) \neq 0, u_{0}, u_{1} \in D(A)$. Then solution of problem (12)-(14) exists and it is unique.

Proof. Since $u_{0} \in D(A)$ and every function of the self-adjoint operator $A$ commutes with $A$, then the element $E_{\alpha, \alpha}\left(T^{\alpha} A\right) u_{0}$ belongs to $D(A)$. Since $u_{1} \in D(A)$ then $h \in D(A)$. It follows directly from the definition of the Mittag-Leffler function that

$$
s^{\alpha-1} E_{\alpha, \alpha}\left(z s^{\alpha}\right)=\frac{d}{d s}\left[s^{\alpha} E_{\alpha, \alpha+1}\left(z s^{\alpha}\right)\right] .
$$

Integrating (15) by parts, we obtain

$$
\begin{gathered}
F(z)=\int_{0}^{T}(T-s)^{\alpha-1} E_{\alpha, \alpha}\left(z(T-s)^{\alpha}\right) \varphi(s) d s=\int_{0}^{T} s^{\alpha-1} E_{\alpha, \alpha}\left(z s^{\alpha}\right) \varphi(T-s) d s= \\
=\int_{0}^{T} \varphi(T-s) d\left[s^{\alpha} E_{\alpha, \alpha+1}\left(z s^{\alpha}\right)+\frac{1}{z}\right] d s=\left.\varphi(T-s)\left[s^{\alpha} E_{\alpha, \alpha+1}\left(z s^{\alpha}\right)+\frac{1}{z}\right]\right|_{0} ^{T}+ \\
+\int_{0}^{T}\left[s^{\alpha} E_{\alpha, \alpha+1}\left(z s^{\alpha}\right)+\frac{1}{z}\right] \varphi^{\prime}(T-s) d s=\varphi(0)\left[T^{\alpha} E_{\alpha, \alpha+1}\left(z T^{\alpha}\right)+\frac{1}{z}\right]-\frac{\varphi(T)}{z}+ \\
+\int_{0}^{T}\left[s^{\alpha} E_{\alpha, \alpha+1}\left(z s^{\alpha}\right)+\frac{1}{z}\right] \varphi^{\prime}(T-s) d s .
\end{gathered}
$$

Let us consider first and last terms in the right hand side of (19). 
It is known ( $[24$, p. 12] $)$ that when $0<\alpha<1$

$$
E_{\alpha, \beta}(z)=-\frac{1}{\Gamma(\beta-\alpha) z}+O\left(|z|^{-2}\right) \quad(z \in R, z \rightarrow-\infty) .
$$

Then for $z \rightarrow-\infty$ we have

$$
E_{\alpha, \alpha+1}(z)=-\frac{1}{z}+O\left(|z|^{-2}\right) .
$$

From the last relation we obtain

$$
T^{\alpha} E_{\alpha, \alpha+1}\left(z T^{\alpha}\right)=T^{\alpha}\left(-\frac{1}{z T^{\alpha}}+O\left(1 /\left(z T^{\alpha}\right)^{2}\right)\right)=-\frac{1}{z}+O\left(1 / z^{2}\right)
$$

when $z \in R, z \rightarrow-\infty$. Thus

$$
\varphi(0)\left[T^{\alpha} E_{\alpha, \alpha+1}\left(z T^{\alpha}\right)+\frac{1}{z}\right]=O\left(1 / z^{2}\right) .
$$

It follows from (20) that for sufficiently large negative values of $z$

$$
\left|E_{\alpha, \alpha+1}\left(z s^{\alpha}\right)+\frac{1}{z s^{\alpha}}\right| \leqslant \frac{C}{\left(z s^{\alpha}\right)^{2}},
$$

where constant $C$ does not depend on $s$. Multiplying the last inequality by $s^{\alpha}$ we obtain the following estimate

$$
\left|s^{\alpha} E_{\alpha, \alpha+1}\left(z s^{\alpha}\right)+\frac{1}{z}\right| \leqslant \frac{C}{z^{2} s^{\alpha}},
$$

and we have

$$
\left|\int_{0}^{T}\left[s^{\alpha} E_{\alpha, \alpha+1}\left(z s^{\alpha}\right)+\frac{1}{z}\right] \varphi^{\prime}(T-s) d s\right| \leqslant \frac{C}{z^{2}} \int_{0}^{T} \frac{\left|\varphi^{\prime}(T-s)\right|}{s^{\alpha}} d s=\frac{C}{z^{2}} \int_{0}^{T} \frac{\left|\varphi^{\prime}(s)\right|}{(T-s)^{\alpha}} d s .
$$

According to the theorem condition, the function $\left|\varphi^{\prime}(s)\right|$ is integrable at zero and the integrand in the last integral is equivalent to $\left|\varphi^{\prime}(s)\right| / T^{\alpha}$. Hence the integral converges at zero. For $s \rightarrow T$ the same function is equivalent to $\left|\varphi^{\prime}(T)\right| /(T-s)^{\alpha}$. Since $\alpha<1$ then the integral also converges at $s=T$.

It follows from (21), (22) that the characteristic function

$$
F(z) \sim-\frac{\varphi(T)}{z}, \quad z \in R, z \rightarrow-\infty .
$$

Let us use now the results presented in [24]. It follows from reviews of Chapter 4 of this work (theorem 4.4.1, p.101) that when $0<\alpha<1$ and $\beta \geqslant \alpha$ the Mittag-Leffler function $E_{\alpha, \beta}(z)>0$ when $z \leqslant 0$. Therefore, it follows from conditions of the theorem that characteristic function (15) has no zeros on the real semiaxis $z \leqslant 0$. It follows from the calculus of self-adjoint operators that operator $B=F(A)$ is invertible. We obtain from the asymptotic equality (23) that domain of the operator

$$
B^{-1}=\int_{-\infty}^{0} \frac{d E_{\lambda}}{F(\lambda)}
$$


coincides with $D(A)$. The element $h$ is defined by formula $(17)$ and it belongs to $D(A)$ then equation (18) has the unique solution $p=B^{-1} h$ and function $u(t)$ is defined by equality (11) where one should set $f(t)=\varphi(t) p$. This completes the proof of theorem 2 .

Let us consider the model case $\varphi(t)=t^{\beta}$. The conditions of theorem 2 for this case are valid when $\beta \geqslant 0$. Moreover, in this case one can write the characteristic function explicitly. Performing the substitution $s=T \xi$ in (15), we obtain

$$
\begin{gathered}
F(z)=\int_{0}^{T}(T-s)^{\alpha-1} E_{\alpha, \alpha}\left(z(T-s)^{\alpha}\right) s^{\beta} d s=\int_{0}^{T}(T-s)^{\alpha-1} s^{\beta} \sum_{n=0}^{\infty} \frac{\left(z(T-s)^{\alpha}\right)^{n}}{\Gamma(\alpha n+\alpha)} d s= \\
=\sum_{n=0}^{\infty} \frac{z^{n}}{\Gamma(\alpha n+\alpha)} \int_{0}^{T}(T-s)^{\alpha n+\alpha-1} s^{\beta} d s=\sum_{n=0}^{\infty} \frac{z^{n} T^{\alpha n+\alpha+\beta}}{\Gamma(\alpha n+\alpha)} \int_{0}^{1} \xi^{\beta}(1-\xi)^{\alpha n+\alpha-1} d \xi= \\
=\sum_{n=0}^{\infty} \frac{z^{n} T^{\alpha n+\alpha+\beta}}{\Gamma(\alpha n+\alpha)} B(\beta+1, \alpha n+\alpha)=\sum_{n=0}^{\infty} \frac{z^{n} T^{\alpha n+\alpha+\beta}}{\Gamma(\alpha n+\alpha)} \cdot \frac{\Gamma(\beta+1) \Gamma(\alpha n+\alpha)}{\Gamma(\alpha n+\alpha+\beta+1)}= \\
=\Gamma(\beta+1) T^{\alpha+\beta} \sum_{n=0}^{\infty} \frac{\left(z T^{\alpha}\right)^{n}}{\Gamma(\alpha n+\alpha+\beta+1)}=\Gamma(\beta+1) T^{\alpha+\beta} E_{\alpha, \alpha+\beta+1}\left(z T^{\alpha}\right) .
\end{gathered}
$$

It follows from the above-mentioned properties of the Mittag-Leffler function that characteristic function (24) is positive on the negative semiaxis and it has asymptotic bound const/z at $-\infty$. Hence it follows that operator $B$ is invertible on $D(A)$.

Corollary 1. Let us assume that $H$ is a Hilbert space, operator $A$ is self-adjoint and non-positive, $0<\alpha<1, \beta \geqslant 0$ and elements $u_{0}, u_{1} \in D(A)$. Then the solution of problem

$$
\begin{gathered}
D^{\alpha} u(t)=A u(t)+t^{\beta} p, t>0, \\
\lim _{t \rightarrow 0} D^{\alpha-1} u(t)=u_{0}, \\
u(T)=u_{1} .
\end{gathered}
$$

exists, it is unique and has the form

$$
\begin{aligned}
& p=\frac{1}{\Gamma(\beta+1) T^{\alpha+\beta}} E_{\alpha, \alpha+\beta+1}^{-1}\left(T^{\alpha} A\right)\left(u_{1}-T^{\alpha-1} E_{\alpha, \alpha}\left(T^{\alpha} A\right) u_{0}\right) \\
& u(t)=t^{\alpha-1} E_{\alpha, \alpha}\left(t^{\alpha} A\right) u_{0}+\int_{0}^{t}(t-s)^{\alpha-1} E_{\alpha, \alpha}\left((t-s)^{\alpha} A\right) s^{\beta} p d s .
\end{aligned}
$$

Remark 1. The last formula can be simplified. Let us note that equality (11) can be written in the form

$$
u(t)=t^{\alpha-1} E_{\alpha, \alpha}\left(t^{\alpha} A\right) u_{0}+F_{t}(A) p,
$$

where $F_{t}(z)$ is obtained from characteristic function (15) by replacing $T$ with $t$. Since in our particular case $F(z)=\Gamma(\beta+1) T^{\alpha+\beta} E_{\alpha, \alpha+\beta+1}\left(z T^{\alpha}\right)$, then function

$$
F_{t}(z)=\Gamma(\beta+1) t^{\alpha+\beta} E_{\alpha, \alpha+\beta+1}\left(z t^{\alpha}\right)
$$

and

$$
u(t)=t^{\alpha-1} E_{\alpha, \alpha}\left(t^{\alpha} A\right) u_{0}+\Gamma(\beta+1) t^{\alpha+\beta} E_{\alpha, \alpha+\beta+1}\left(t^{\alpha} A\right) p
$$

We should also mention the case $\beta=0$. The study of inverse problems of the type considered here began with this option (see [14-16]). 
Corollary 2. Let us assume that $H$ is a Hilbert space, operator $A$ is self-adjoint and non-positive, $0<\alpha<1$, elements $u_{0}, u_{1} \in D(A)$. Then the solution of problem

$$
\begin{gathered}
D^{\alpha} u(t)=A u(t)+p, t>0, \\
\lim _{t \rightarrow 0} D^{\alpha-1} u(t)=u_{0}, \\
u(T)=u_{1}
\end{gathered}
$$

exists, it is unique and has the form

$$
\begin{gathered}
p=\frac{1}{T^{\alpha}} E_{\alpha, \alpha+1}^{-1}\left(T^{\alpha} A\right)\left(u_{1}-T^{\alpha-1} E_{\alpha, \alpha}\left(T^{\alpha} A\right) u_{0}\right), \\
u(t)=t^{\alpha-1} E_{\alpha, \alpha}\left(t^{\alpha} A\right) u_{0}+t^{\alpha} E_{\alpha, \alpha+1}\left(t^{\alpha} A\right) p .
\end{gathered}
$$

\section{References}

[1] S.G.Samko, A.A.Kilbas, O.I.Marichev, Fractional Integrals and Derivatives. Theory and Application, Gordon and Breach Science Publishers, 1993.

[2] K.S.Miller, B.Ross, An Introduction to the Fractional Calculus and Fractional Differential Equations, A Wiley-Interscience Publication, John Wiley and Sons, Inc., New York, 1993.

[3] A.M.Nakhushev, Fractional calculus and Applications, Fizmatlit, Moscow, 2003 (in Russian).

[4] A.V.Pskhu, Boundary value problems for partial differential equations fractional and continual order, Nalchik, 2005 (in Russian).

[5] A.N.Kochubei, The Cauchy problem for evolution equations of fractional order, Differentsial'nye uravneniya, 25(1989), no. 8, 1359-1368 (in Russian).

[6] V.A.Kostin, The Cauchy problem for an abstract differential equation with fractional derivatives, Dokl. Akad. Nauk SSSR, 326(1992), no. 4, 597-600 (in Russian).

[7] A.V.Glushak, The Cauchy type problem for abstract differential equation with fractional derivative, Vestnik Voronezh. Gos. Univ., Fiz.-mat., 2001, no, 2, 74-77 (in Russian).

[8] Ph.Clement, G.Gripenberg, S.-O.Londen, Regularity properties of solutions of fractional evolution equation. Evolution equations and their application in physical and life science, Lecture Notes in Pure and Appl. Math., 215(2001), Dekker, New York, 235-246.

[9] H.K.Awad, A.V.Glushak, On a perturbation of an abstract differential equation with fractional derivatives by nonlinear operator. Sovremennaya matematika. Fundamental'nye napravleniya, 35(2010), 5-21 (in Russian).

[10] K.Yosida, Functional Analysis, Springer-Verlag, 1980.

[11] A.V.Glushak, On a perturbation of an abstract differential equation with Riemann-Liouville fractional derivatives. Differentsial'nye uravneniya, 46(2010), no. 6, 859-873 (in Russian).

[12] A.I.Prilepko, Inverse problems of Potential theory (elliptic, parabolic, hyperbolic equations and transport equation), Matematicheskie zametki, 14(1973), no. 6, 755-767 (in Russian). 
[13] A.I.Prilepko, D.G.Orlovsky, I.A.Vasin, Methods for solving inverse problems in mathematical physics, New York, Basel, Marcel Dekker, 2000.

[14] A.D.Iskenderov, R.G.Tagiev, The inverse problem of determining the right parts of evolution equations in Banach space, Nauchnyye Trudy Azerbaidzhanskogo Gosudarstvennogo Universiteta, 1979, no. 1, 51-56 (in Russian).

[15] W.Rundell, Determination of an unknown nonhomogeneous term in a linear partial differential equation from overspecified boundary data. Appl. Anal., 10(1980), 231-242.

[16] Yu.S.Eidelman, Two-point problem for a differential equation with a parameter, Dokl. Akad. Nauk UkrSSR, ser. A, 1983, no. 4, 15-18 (in Russian).

[17] I.V.Tikhonov, Yu.S.Eidelman, The inverse problem for differerential equation in a Banach space and the distribution of zeros of entire the Mittag-Leffler function. Differentsial'nye uravneniya, 38(2002), no. 5, 637-644 (in Russian).

[18] D.G.Orlovsky, One inverse problem for the equation of Maxwell-Boltzmann Journal of Siberian Federal University. Mathematics and Physics, 2(2009), no. 3, 328-336 (in Russian).

[19] D.G.Orlovsky, Fredholm solvability of boundary inverse problems for abstract differential equations second order, Differentsial'nye uravneniya, 28(1992), no. 4, 687-697 (in Russian).

[20] M.M.Kokurin, The uniqueness of a solution to the inverse Cauchy problem for a fractional differential equation in a Banach space, Russian Math. (Iz. VUZ), 57(2013), no. 12, 16-30.

[21] M.M.El-Borai, On the solvability of an inverse fractional abstract Cauchy problem, Int. J. of Research and Review in Applied Science, 4(2010), no. 4, 411-416.

[22] A.V.Glushak, Inverse problem for evolution equation with integral of fractional order at boundary condition. Sovremennaya matematika. Fundamental'nye napravleniya, 29(2008), 49-61 (in Russian).

[23] D.G.Orlovsky, to the problem of determining the parameter of the evolution equation Differential'nye uravneniya, 26(1990), no. 9, 1614-1621 (in Russian).

[24] A.Yu.Popov, A.M.Sedletskyi, The distribution of roots of the Mittag-Leffler functions, Sovremennaya matematika. Fundamental'nye napravleniya, 40(2011), 3-171 (in Russian).

\section{Определение параметра дифференциального уравнения дробного порядка с производной Римана-Лиувилля в гильбертовом пространстве}

\section{Дмитрий Г. Орловский}

В гилъбертовом пространстве рассмотрена задача типа Коши для дифференциалъного уравнения с дробной производной и самосопряженным оператором. Поставлена задача определения параметра в неоднородном члене уравнения по значению решения в фиксированной точке. Доказаны теоремы существования и единственности решения.

Ключевые слова: уравнение дробного порядка, гилъбертово пространство, самосопряженный оператор, задача типа Коши, функция Миттаг-Леффлера, обратная задача, характеристическая функиия. 